\title{
Understanding the role of trust in network-based responses to disaster management and climate change adaptation in the Asia-Pacific region
}

\section{Authors}

\section{Victoria L. Ross}

Smart Water Research Centre, Griffith University, Gold Coast Campus, Southport, 4215, Queensland, Australia.

Phone: +61 755527808

Fax: + 61755527831

Email: victoria.ross@griffith.edu.au

\section{Elizabeth L. Malone}

Pacific Northwest National Laboratory, Joint Global Climate Change Institute, MD 20740, United States.

Email: e.malone@pnnl.gov

Susan Kinnear

Central Queensland University, Rockhampton, 4700, Australia.

Email: s.kinnear@cqu.edu.au

\section{Key words}

Disaster management, social networks, trust, social identity theory, cultural cognition theory

\begin{abstract}
Effective communication and strong collaboration amongst organisations are essential tools for disaster management responses and climate change adaptation. Complex situations such as disaster preparation and response require trust-based and collaborative networks. Given that mutual trust and willingness to share information are central to successful disaster management, it is crucial to increase our understanding of how to build and sustain network trust and collaboration. Based on a comprehensive literature review, including both theoretical and empirical studies, we explore the role and impact of trust on facilitating improved network co-ordination in emergency responses to climate-driven events. Social identity theory and cultural cognition theory are applied as a framework for understanding trust development, particularly in the context of the Asia-Pacific region. The paper identifies obstacles to trust development, and then outlines key methods by which trust can be established and enhanced to improve communication and network effectiveness in disaster management. These findings provide critical information for policy makers and network participants with regard to building network trust to better underpin disaster management and climate change adaptation in the Asia-Pacific region.
\end{abstract}

\section{Introduction}

Climate change is predicted to increase the frequency, severity and intensity of natural disasters in the decades to come (Djalante, 2013; WMO, 2014). Globally, the increasing incidence of extreme events presents a significant threat to human society as well as the built environment (Cadag \& Gaillard, 2012; Hidayat \& Egbu, 2010; Kostoulas, Aldunate, Pena 
Mora \& Lakhera, 2008), with casualties and damage to vital infrastructure now being a major global humanitarian concern (Haque, 2003). This is a particularly vexed issue in the the Asia-Pacific region, given that the area currently experiences over 70 percent of the world's natural disasters; and scientists have warned that the intensity and/or frequency of disasters in the region are likely to increase further (APEC, 2014; Haque, 2003). The recent IPCC fifth assessment report (AR5) noted that in the Asia region, extremes of precipitation will be characterised by strong variability, with both increasing and decreasing trends being observed spatially across Asia, and temporally across seasons (IPCC, 2014a). The IPCC also reports a high degree of confidence that 'extreme climate events will have an increasing impact on human health, security, livelihoods and poverty; (IPCC, 2014a, p.3).

Given the clear link between climate change, disaster vulnerability and disaster management (Bijay, Leal Filho \& Schulte, 2013), disaster response and mitigation are becoming increasingly important as strategies for adaptation to climate change and regional sustainability (Malone \& Kinnear, 2014). Better preparation for, and responses to, emergencies and disasters are a priority across the Asia-Pacific, as this helps to reduce the risk of disasters and creates the ability to build business and community resilience (APEC, 2014). However, tackling this agenda is complex and multifaceted, reaching across multiple tiers of government, communities, organisations and individuals.

This paper is innovative in exploring the impact of trust on facilitating improved network coordination in emergency responses, particularly in the Asia Pacific region. Existing research has demonstrated the link between effective response and recovery activities and wellcoordinated, inter-organisational networks and trust (Cadag \& Gailard, 2012; Kapucu, 2005). However, no studies to date have established how and why this occurs, and what the implications are for those organisations working in climate change adaptation. In this paper, a theoretical framework is presented to provide an understanding of trust development, obstacles to trust are identified, followed by an outline of strategies by which trust can be established and enhanced to improve communication and network effectiveness in disaster management. Finally, practical recommendations for trust building strategies are provided for disaster management network managers. In applying these, however, it must be acknowledged that this review is constrained by the available literature on social networks and trust, particularly the paucity of literature that examines these concepts using field examples from the Asia-Pacific. The understanding and measurement of network-based trust is a nascent field, and as such this paper is an exploratory, and offers a review of insights-todate in this developing field.

\section{Understanding trust and networks - an historical perspective}

Trust and networks have been studied for decades as elements of social capital, economic transactions, and organizational performance. Jane Jacobs emphasized the important role trust plays the dense urban networks that constitute social capital (Jacobs, 1961). Trust among the shopkeepers and residents of neighborhoods meant that people networks effectively addressed problems such as crime, whereas the lack of trust among groups in the Hausmannstyle "City Beautiful" effectively prevented social capital of this type, instead fostering distrust among groups estranged from each other, necessitating strong police forces and other formal organizations. These themes were developed with regard to business development by ethnic groups in the 1970s (see Fukuyama, 1997 for this history). Later, sociologists in the 1980s and 1990s, notably Robert Putnam (1993, 1995) emphasized trust as a necessary component of the shared values that promote or prevent economic prosperity. 
Trust, of course, may facilitate action within a group but hinder actions that must be taken with outsiders; that is, trust may be based on strong, shared values that are the very core of the organization and thus include distrust of outsiders who, by definition, do not share those values (Fukuyama 1995). This is the dilemma of organizations that are joined in a network without any or much of a basis for trust among the organizations. Their "radius of trust" may not extend beyond their own organizations, so trust needs to be consciously built. Unfortunately, the theoretical and empirical work on trust and networks does not examine the case of organizations that are directed to work together, i.e., form a network to perform specific tasks or functions. Rather, research and analysis has been targeted at firms who voluntarily work together, knowledge networks in the case of research groups (e.g., Hardwig 1991), or at face-to-face networks (families, kin, ethnic groups, civil society organizations). This leaves a conspicuous gap in the literature, particularly in the context of disaster management, which typically calls for numerous organisations to co-deliver responses to acute climate events.

\section{Understanding trust and collaborative networks in disaster management}

To achieve a rapid and effective response to unexpected events, multi-agency disaster management is often comprised of collaboration amongst geographically-distributed organisations, both public and private (Jansson, Lee, Bharosa \& Cresswell, 2010). Within this, a key challenge for disaster relief efforts is the need for these numerous organisations to collaborate effectively, despite often having had no previous interactions with one another (Kapucu \& Garayev, 2011; Kostoulas et al., 2006). Furthermore, participating organisations often have qualitatively different ideas about how collaboration should work and what elements and outcomes of disaster relief are the most important (Malone \& Kinnear, 2014). Not surprisingly, research studies have thus shown that inter-organizational communication is not always effective during a crisis (Kinnear et al., 2013; Longstaff \& Yang, 2008; Palttala, Boano, Lund \& Vos, 2012; Moe \& Pathranarakul, 2006).

Palttala et al. (2012) suggest that differing organisational cultures, structures and approaches are a hindrance to cooperation during a crisis, although, at least theoretically, these differences could also complement each other and provide a basis for more comprehensive responses. Jansson et al. (2010) stated that many disaster management systems lack the capacity to cope with complexity and uncertainty; possibly driven by a lack of human-centred approaches to improve multi-agency disaster management. In particular, trust-based relationships have been identified as critical for effective emergency responses (Jansson et al., 2010; Kapucu, 2005; Kapucu \& Garayev, 2011; Longstaff \& Yang, 2008).

Why do complex situations such as disaster response and recovery require trust-based and collaborative networks? A strong body of research has demonstrated the important role of trust in network collaboration, communication and co-operation (e.g., Beratan, 2007; Fisher, 2013; Janssen et al., 2010; Kapucu \& Garayev, 2011; Longstaff \& Yang, 2008; Moe \& Pathranarakul, 2006; Vasavada, 2013). In interpreting this material, it is worthwhile to note that trust is important between emergency responders; but so too it is important for those organisations to be trusted by the public they serve (Longstaff \& Yang, 2008). According to Beratan (2007), it is necessary to establish an interpersonal culture of trust and pragmatism in order that collaborative social networks can operate effectively. Trust is also critical for network performance and sustainability (Provan \& Kenis, 2008), and has been demonstrated to accelerate coordination in emergency management response operations (Kapucu, 2005). 
Longstaff and Yang (2008) conducted research to test the role of trust in the planning and management of communication in crisis situations; and concluded that trust is one of the most important variables in effective communication management in times of "surprise."

The authors analysed reports from a wide variety of global crisis incidents and found a direct correlation between trust and the level of an organization's preparedness and internal coordination of crisis communication. This provided strong evidence that a higher level of trust led to better internal coordination of crisis communications with the organisation's stakeholders as well as with external media. However, the authors stressed that trust must be two-directional (i.e., "I will trust local government if it trusts me enough to be prepared to tell me the whole story."). Their findings also indicated that more pre-crisis preparedness can lead to significantly less attribution of blame to other parties during and after a crisis event.

While network structures are often hierarchical (government bureaucracy, for example), the dynamic situations presented by disaster responses actually require the simultaneous operation of hierarchies as well as decentralised nodes, in order to facilitate an effective response. Here, trust-based networks are preferable to other network architectures, including those based on market mechanisms (which do not function in disaster response, except possibly in a predatory way) and those based on regulations and sanctions (which may be difficult to enforce and inadequate for disaster conditions) (Gausdal \& Hildrum, 2012).

\section{Trust and disaster management in the Asia Pacific region}

A paucity of research exists regarding the role of trust in disaster management specifically within the Asia Pacific region. However, several key research papers relevant to the region are outlined below.

Moe and Pathranarakul (2006) conducted a detailed case study of Thailand's tsunami responses, examining the critical success factors for successful disaster management. They argued that many developing countries prone to natural disasters appear to lack proactive strategies for early warning, mitigation and preparedness. Moe and Pathranarakul reported that project success was strongly linked to communication and co-operation between stakeholders, and concluded that trust facilitated by effective communication between key stakeholders was the key factor in successful disaster management (Moe \& Pathranarakul, 2006).

According to Nakagawa and Shaw (2004), broader social capital (comprising trust, social norms and networks) also plays an important role in disaster recovery. These scholars examined the role of social capital in the post-earthquake rehabilitation and reconstruction programs across two case study regions: Kobe, Japan and Gujarat, India. Despite differences in socio-economic and cultural contexts, the authors demonstrated that the social capital elements of trust, social norms and networks were positively associated with the highest satisfaction rates with post-disaster planning as well as the fastest recovery time (Nakagawa \& Shaw, 2004).

Consistent with these findings, Vasavada (2013) asserts that high levels of trust are a crucial mechanism for effective networked responses for disaster management. Vasavada studied a disaster management network in the state of Gujarat, India. Due to its geography, Gujarat has experienced numerous natural disasters, and its underdeveloped infrastructure makes the area particularly vulnerable to the effects of these events. Using social network analysis and 
interviews, the research examined the structure of the disaster management network and identified the key factors for network effectiveness: these included trust; the number of participants in the network; goal consensus and the need for network-level competencies. Results showed a high density of trust among the central actors who shared an interdependence for financial resources and outreach efforts; there were also higher levels of trust among peripheral actors who had worked together prior to the disaster and thus had preestablished relationships. By contrast, peripheral actors had a low level of trust in the central actors. Vasavada stressed that it was important for network members to focus on trust and goal consensus as being necessary to underpin effective recovery efforts; as these factors are particularly helpful in managing the dynamics of the network relationships.

In Australia, Kinnear et al. (2013) conducted research on network governance and climate change adaption in response to the widespread 2010/2011 Queensland floods, which affected 70 percent of the State and had large personal and social impacts (Kinnear et al., 2013). Primary data collected from organisations involved in disaster management and water management were analysed to examine collaboration and communication patterns; changes in the network structure from routine management to flood operations; similarities and differences between the geographic regions, and whether collaboration was correlated with trust. Results showed that having trust in other organisations and using personal networks to establish good working relationships were highly valued by respondents. High value was also placed on collaborators who were experienced, flexible, trustworthy, and had good knowledge and understanding of local contexts. Providing and receiving resources and information tended to be associated with trust, echoing the finding of Vasavada (2013). Some of the key implications for policy and practice included the need for stakeholders to drive adaptation to climate change through collaboration and communication; the need for stakeholders to share a common goal and language; and the need to establish collaboration outside of disaster events (Kinnear et al., 2013).

Taken together, this series of research studies from the Asia Pacific region show that trust is essential for establishing good working relationships, and in turn, effective social networks in disaster management. It is likely that trust is so important in this context because people (and hence, organisations) have an inherent desire for human-centred approaches and for interpersonal relationships when coping with complexity and uncertainty. In considering this, the theoretical framework described in the next section provides an understanding of peoples' needs for social relationships to reduce perceptions of risk and generate trust.

\section{A theoretical framework for understanding trust}

Given the critical importance of trust to effective disaster response operations (Jansson et al., 2010; Kapucu, 2005; Kapucu \& Garayev, 2011; Longstaff \& Yang, 2008), it is crucial to increase the current understanding of how to build and maintain network trust and collaboration. Examination of the trust literature shows that definitions of trust vary widely (Twyman, Harvey \& Harries, 2008). Notwithstanding this, the most common elements for the existence of trust appear to be, vulnerability, risk, and positive expectations regarding the behaviour of others. A widely accepted definition of trust is 'a psychological state comprising the intention to accept vulnerability based upon positive expectations of the intentions or behaviour of another' (McEvily, Perrone \& Zaheer, 2003; Rousseau, Sitkin, Burt \& Camerer, 1998). According to researchers, personal vulnerability lies at the heart of trust - if we did not feel vulnerable, we would not feel the need to trust people (e.g., Hogg, 2007; Marar, 2003). 
Two contrasting orientations have been advanced to explain the development of trust. The instrumental or rational model views trust as a subjective calculation of the potential costs and benefits of future interactions. However, this approach has been criticised for affording too little role to emotional and social influences on trust decisions (Kramer, 1999). In contrast, the relational model emphasises social identification and social relationships in relation to trust (Tyler \& Degoey, 1996). The relational framing of trust suggests that trust is related to the nature of social bonds and shared identities between group members (Tyler \& Degoey, 1996). According to this perspective, shared social identification (a sense of "we"ness, or belonging to a social group) enhances trust in other community members, thus increasing cooperative behaviour (Tyler \& Degoey, 1995). The relational model of trust provides a valuable framework for understanding why trust appears to be linked to peoples' needs for social relationships. It is particularly useful when applied to broader social network analysis, which is increasingly being used to examine relationships amongst actors in disaster management settings.

The relational framing of trust accords with social identity theory, which posits that social groups provide members with a social identity: a definition of whom one is and an evaluation of what that entails (Hogg \& Abrams, 1998; Tajfel \& Turner, 1986). Research from the social identity perspective has shown that people who belong to our groups (i.e., in-group members) are perceived in more desirable ways - such as being more trustworthy or honest - compared with outsiders; and that shared social identity reduces risk and generates in-group trust (Brewer, 2007; Hogg, 2007; Ross, Fielding \& Louis, 2014; Tanis \& Postmes, 2005). According to Hogg (2007), we trust fellow group members to do us no harm if they are "one of us" and behave in ways that protect and promote the group. However, group trust also features an inter-group dimension, where members of other groups can be viewed with suspicion and distrust (Hogg, 2007).

Within the context of disaster management in the Asia-Pacific, and particularly in the case of networked governance studies, social identity theory can help us understand why distrust may exist between different organisations. This also raises the interesting phenomenon of some organisations essentially being perceived as 'out-group' although they are in fact actors within the social network, and may record a high density of social ties. As will be discussed in a later section, social identity theory can also be applied to develop methods to build trust between the different organisations involved in disaster management.

Another theory that recognises the influence of social and cultural factors on trust and risk perceptions is that of cultural cognition. According to this theory, when people have to make decisions in an area in which they do not have expertise, their only option is to rely on experts in whom they trust (Kahan \& Braman, 2006). Here, the trusted people are inevitably ones whose cultural values they share, and who are inclined to credit or dismiss scientific evidence based on its conformity to their cultural values. In other words, cultural cognition influences perceptions of credibility (Kahan, Jenkins-Smith, \& Braham, 2011).

According to cultural cognition theory, individuals reflexively reject information that is inconsistent with their predispositions, especially when they perceive that it is being advocated by experts whose values they reject and opposed by alternative people whose values they share. Kahan et al. therefore assert that to overcome the cultural cognition effect, communicators must attend to the cultural meaning of information, and take care when 'framing' messages for intended audiences. Here, framing needs to consider that people 
interpret information through their ideological filters and are strongly influenced by their own identity, worldview, and belief systems (Kahan, et al., 2011).

The approaches outlined above share a commonality in suggesting that people are not always 'rational' in their perceptions and decision-making due to the influence of perceptual biases. Rather, all decisions are made through a complex process of emotion, personal and social filtering. Audiences filter information through the lenses of their value and belief systems, as well as cultural experiences and norms (Vaughan \& Hogg, 1995). This is an important message given that all organisations are comprised of people, each bringing their own dynamics. Hence, it is reasonable to conclude that communication amongst people and organisations will need to be complex and nuanced, if the development of trust is to be fully realised.

\section{Obstacles to trust development}

Establishing trust is a major challenge in extreme situations due to the involvement of diverse response teams and the frequently changing actors that emergency responders have to deal with during disasters (Kapucu \& Garayev, 2011; Kostoulas et al., 2008). According to Kostoulas et al. (2008), first responders can be hesitant to communicate and interact with those outside of their organization due to a lack of prior interactions. As foreshadowed earlier, this tendency to be distrustful of those outside of one's group is consistent with social identity theory and the concept of inter-group suspicion and distrust. Kostoulas et al. (2008) assert that it is the task of first responders to assess the trustworthiness of others in order to reduce uncertainty. However, there is often considerable pressure to do this quickly, which may distort the otherwise gradual sequence of trust development. For example, according to Beratan (2007), trust has to be earned over time, through repeated experience of positive interaction.

It is not yet clear whether trust-connections built at unnaturally rapid speed are more tenuous compared than those grown gradually; or if they in fact have a similar durability once formed, but that the likelihood of genuine formation is decreased under time-pressured circumstances. What is clear, however, is that organisations tend to be reluctant to commit to relationship and trust-building activities because of the time imposition, the possibility of failure, and the difficulties in recognising progress (Beratan, 2007). The latter is an especially acute problem because it is difficult to measure the level of trust among participating organizations during emergencies, especially where different and frequently-changing actors are involved (Kapucu \& Garayev, 2011). In the absence of an agreed metric, this may lead to nonchalance amongst network actors with regard to the basic value of trust, and less propensity to invest in building trust assets. For example, Beratan (2007) claims that few organisations, particularly public sector agencies, feel that they can afford to persist with activities that do not produce visible short-term progress. However, in the Asia Pacific region, where climate events are sudden, and where there may be rapid 'churn' of management in government and/or other key disaster response organisations, it could be argued a time-lag to allow for trust development is a luxury that cannot often be afforded; thus intensifying the need to dedicate resources to trust development in the pre-preparation phase.

The second key barrier to trust-building is the tendency for organisations to have differing cultures, structures and approaches. The many different groups of responders involved in relief operations (e.g., police officers, firefighters, medical personnel, civil engineers, public and private agencies) have distinct roles, skills, knowledge and experience, as well as policies 
and protocols on how to respond to emergency situations (Kostoulas et al., 2008). Palttala et al. (2012) claim that communication problems occur when actors need to cooperate, but are in fact from organisations that have differing ways of handling a crisis. Given that cultures develop slowly, changing these in favour of greater integration can be a challenge (Palttala et al., 2012).

\section{Building trust}

The adage "'trust arrives on foot but leaves on horseback" by the nineteenth century Dutch politician, Thorbecke, aptly describes the concept of trust being difficult to earn and easy to lose (Nooteboom, 2002). It is well established that trust building is a long-term and time consuming process (Beratan, 2007; de Bruijn \& ten Heuvelhof, 2008; Longstaff \& Yang, 2008; Tummers, Steijn, \& Beckers, 2012; Vasavada, 2013). Within the context of disaster management, Longstaff \& Yang (2008) assert that trust cannot simply be established on the first day of a disaster. Trust has to be earned over time, and can occur only if there is a longterm commitment to the process. Moreover, the collaborative engagement needed for trust building requires a sincere and visible commitment of time and resources (Beratan, 2007). An interesting question amongst this is the impact that repeated, and more frequent, disaster events may have on the trust dynamics of response organisations across the Asia-Pacific: one possibility is that organisations will become more comfortable and familiar with each other after having partnered on several disaster events. Equally, it may be that organisational values become more fluid, and individuals become more accepting of those from different cultural bias. On the other hand, it is also possible that trust may break down over time, because of negative experiences in working with others, particularly if those organisations are poorly prepared or ineffective in communicating.

Longstaff and Yang (2008) assert that perhaps the most important and least understood role for policy makers is insuring that emergency communications can be trusted by other emergency responders and by the public. Longstaff and Yang suggest that as a minimum, ongoing communication should be provided to help build trust; accompanied by mandated communications plans to guide organisational responses in times of surprise. Organisations that have previously been involved in collaborative efforts with dialogue between actors appear to have a greater ability to build trust (Ansell \& Gash, 2008; Kapucu \& Garayev, 2011). Gaillard (2010) states that a concerted effort is needed to open dialogue and thus build trust between scientists, non-governmental organisations, disaster reduction practitioners and local communities. Palttala et al. (2012) also found that organizations with a good reputation are considered trustworthy, and other actors are willing to share information with these. In a practical vein, technologies such as mobile phones, the internet, geographical information systems, and community radios can help to strengthen networks and promote effective disaster response by keeping those who trust one another in communication (Ospina, 2011).

Trust building in disaster management requires not just professional expertise to rescue people and mitigate harmful consequences, but also openness, empathy, and explaining decisions and alternatives (Palttala et al., 2012). Palttala et al. conducted a study to identify gaps in communication in disaster management; asking experts from governmental organizations and NGOs about their experiences in disaster management and communication. The results stressed the importance of honesty, candour and openness in crisis communications. Clarity is crucial in the construction of crisis messages, as inadequate communication leads to confusion and public mistrust (Palttala et al., 2012; Seeger, 2006). 
Palttala et al. concluded that the use of accurate, widely distributed, and timely information reduces anxiety and strengthens people's sense of self-efficacy. In this way, trust is a result of actions and communication flow throughout all the crisis phases (Longstaff \& Yang, 2008; Palttala et al., 2012).

\section{Building trust in disaster response networks in the Asia-Pacific region}

Unfortunately, within the Asia-Pacific region, the distribution of accurate and timely communications is not always possible. In developing nations, science and predictive abilities may not be as well advanced, meaning accurate information may not always be available. The large and dispersed population of the Asia-Pacific, coupled with poor baseline communications infrastructure in some areas, could also be a hindrance to achieving mass messaging effectively. Lebel (2014) has reported that 'major knowledge-action gaps exist with respect to adaptation to climate change in the Asia-Pacific', due to missing, inaccessible or unused information. In this landscape, it may be very difficult to build trust through accurate and timely communications.

So, how can organisations effectively build trust when many of the factors on which trust development is influenced, are external to their control? Below we outline key recommendations for policy makers and disaster management network participants for facilitating trust building in the Asia Pacific region. These strategies should prove particularly valuable for disaster management network managers.

\section{Recommendations}

\section{Emphasising a common goal and shared identity}

A key strategy for facilitating trust building is through an emphasis on striving to achieve a common goal (i.e., effective disaster response). As discussed previously, social identity theory asserts that we trust others if they are perceived to be a member of our group, or "one of us". Consistent with both social identity theory and cognitive cognition theory, it is wellestablished that we also trust people whose values are compatible with our own goals or are perceived to have shared values (Earle \& Cvetkovich, 1995; Ross et al., 2014; Siegrist, 2007; Vaske, Bright \& Absher, 2007). Therefore, in order to develop a sense of shared social identity and network trust it will be vital for disaster management communicators to emphasise that all organisations are "working together for a common goal". In addition, intergroup suspicions and distrust could be overcome if network organisations perceive themselves as belonging to a larger group of emergency responders all working to an effective disaster response.

As trust is based on the communicator and target persons sharing similar values, Siegrist et al. (2007) stresses that communicators should therefore not only be knowledgeable about the facts of the case, but should primarily be knowledgeable the concerns and values of the target audience. In the context of building network trust in the Asia Pacific region, it will be important to be cognizant of peoples' inherent desire for human-centred approaches when coping with complexity and uncertainty. In this way trust can be facilitated through developing a sense of shared values and concerns, and thus 'we-ness' among disaster response networks. This sense of shared social identification through understanding values of 
network partners will enhance trust and facilitate co-operative behaviour (Tyler \& Degoey, 1995).

\section{Understanding cultural values}

The research reviewed in this chapter shows that people are not necessarily rational in their perceptions and decision-making due to the influence of perceptual biases. Cultural cognition theory explains the tendency of individuals to trust those who they perceive to have shared values, and distrust or reject information from those they perceive to oppose their values (Kahan et al., 2011). In the Asia Pacific region, where there are a wide range of differing cultures, it will be vital for communicators to attend to the cultural meaning of information and messaging in order to overcome the cultural cognition effect. Understanding differences in opinions and working towards building consensus will be central to facilitating (and sustaining) network trust.

\section{Attending to message framing}

When framing messages it should also be considered that people interpret information through their ideological filters and are strongly influenced by their identity, worldview, and belief systems. It will also be important to take into account the cultural values and endpoint goals, as already described above. It will be critical to convey messages that do not directly challenge people's embedded values, but instead encourage consensus building among diverse groups. This approach will be particularly important when establishing network trust in the geographically and culturally diverse expanse of the Asia-Pacific.

\section{Build trust early and repeatedly}

The extant literature clearly shows that trust-building requires an investment of time, yet conversely, climate impacts are typically sudden and acute, leaving little time for the establishment of trust. Hence, the importance of pre-disaster planning and scenario-based training is likely to increase, as the Asia-Pacific confronts the reality of climate change. Furthermore, Fukuyama (1995) categorically states that trust is essential to functioning networks: trust may begin with trust in professional standards, reputation, ethnicity, or some other basis, but it must be fostered for the tasks of the network. Hence, the regular flux (substitution, replacement, or entry/exit) of actors throughout disaster networks also signals the need to commit resources to ensure the sustainability of trust-based partnerships.

\section{Explore metrics for trust}

Future research work in this field may concentrate on identifying both qualitative and quantitative measures of trust. Based on his analysis of social capital and the role of trust, Fukuyama (1997:429) proposed a "radius of trust" measure of social capital, which measures "the degree to which individual members are capable of collective action on the basis of mutual trust." However, a practical application of this theory has not been brought forward: this is a necessary development, as without it, organisations will be without a tangible appreciation of their existing levels of trust, and whether these are being maintained or degraded over time. 


\section{Conclusions}

The Asia Pacific region, with a large and dispersed population and difficulties in distributing accurate and timely communications, presents unique challenges for building network trust. This work makes a significant contribution to the climate change adaptation literature by providing a insight into how network trust can be facilitated, specifically in the Asia pacific region. The relational model of trust, as well as both social identity and cultural cognition theories have provided useful theoretical frameworks for understanding peoples' needs for social relationships to reduce perceptions of risk and generate trust. In applying these models, and integrating the findings of existing case studies from the Asia-Pacific, this review identified at five key learnings to assist future climate change adaptation initiatives: emphasise a common goal and develop shared identity; understand cultural values; attend to message framing; build trust early and repeatedly, and explore the development of metrics for trust, to guide further development of new and existing social networks.

\section{References}

Ansell, C., Gash, A. (2008). Collaborative governance in theory and practice. Journal of Public Administration Research and Theory, 18(4), 543-571.

Asia-Pacific Economic Cooperation (APEC). (2014). Retrieved 5/03/2014. http://www.apec.org/Groups/SOM-Steering-Committee-on-Economic-and-TechnicalCooperation/Working-Groups/Emergency-Preparedness.aspx

Beratan, K. K. (2007). A cognition-based view of decision processes in complex socialecological systems. Ecology and Society, 12(1), 27.

Bijay, P., Leal Filho, W., Schulte, V. (2013). Understanding the links between climate change and disaster management in Pacific Island countries. In Leal Filho, W. (ed.), Climate Change and Disaster Risk Management. Springer-Verlag: Berlin Heidelberg.

Brewer, M.B., (2007). The importance of being we: human nature and intergroup relations. American Psychologist, 62, 728-738.

Cadag, J. R. D., Gaillard, J. (2012). Integrating knowledge and actions in disaster risk reduction: the contribution of participatory mapping. Area, 44, 100-109.

de Bruijn, H., ten Heuvelhof, E. (2008). Management in Networks: On Multi-Actor Decision Making, New York: Routledge.

Djalante, R. (2013). Identifying drivers, barriers and opportunities for integrating disaster risk reduction and climate change adaptation in Indonesia: An analysis based on the Earth system governance framework. In Leal Filho, W. (ed.), Climate Change and Disaster Risk Management. Springer-Verlag: Berlin Heidelberg.

Earle, T.C., Cvetkovich, G.T. (1995). Social Trust: Towards a Cosmopolitan Society. Praeger: London. 
Fisher, R. (2013). 'A gentleman's handshake': The role of social capital and trust in transforming information into usable knowledge. Journal of Rural Studies, 31, 13-22.

Fukuyama, F. (1995). Trust: The Social Virtues and the Creation of Prosperity. Free Press: New York.

Fukuyama, F. (1997). Social Capital: The Tanner Lectures on Human Values. 377-484. Available at http://tannerlectures.utah.edu/_documents/a-to-z/f/Fukuyama98.pdf

Gaillard J.C. (2010). Vulnerability, capacity and resilience: perspectives for climate and development policy. Journal of International Development, 22, 218-232.

Gausdal, A., H., Hildrum, J., M. (2012). Facilitating trust building in networks: a study from the water technology industry. Systemic Practice and Action Research, 25, 15-38.

Haque, C.E., (2003). Perspectives of Natural Disasters in East and South Asia, and the Pacific Island States: Socio-economic Correlates and Needs Assessment. Natural Hazards, 29, 465483.

Hardwig, J. (1991). The role of trust in knowledge. The Journal of Philosophy, 88 (12), 693708.

Hidayat, B., Egbu, C. (2010). A literature review of the role of project management in postdisaster reconstruction. In Egbu, C. (Ed) Procs 26th Annual ARCOM Conference, 6-8 September 2010, Leeds, UK, Association of Researchers in Construction Management, 12691278.

Hogg, M., (2007). Social identity and the group context of trust: managing risk and building trust through belonging. In Siegrist, M., Earle, T.C., Gutscher, H. (Eds.), Trust in Risk Management. Earthscan: London.

Hogg, M.A., Abrams, D. (1998). Social Identification. Routledge: London and New York.

IPCC, (2014a), Working Group II, Fifth Assessment Report, Volume II: Regional Aspects Chapter 24: Asia. Intergovernmental Panel on Climate Change, Accessed online at http://www.ipcc.ch/report/ar5/wg2/

IPCC, (2014b), Working Group II, Fifth Assessment Report, Volume II: Regional Aspects Chapter 25: Australasia. Intergovernmental Panel on Climate Change, Accessed online at http://www.ipcc.ch/report/ar5/wg2/

Jacobs, J. (1961). The Death and Life of Great American Cities. Vintage Books: New York.

Janssen, M., Lee, J., Bahrosa, N., Cresswell, A. (2010). Advances in multi-agency disaster management: key elements in disaster research. Information Systems Frontiers, 12, 1-7.

Kahan, D. M., Braman, D. (2006). Cultural cognition and public policy. Yale Law and Policy Review, 24, 149 - 172. 
Kahan, D.M., Jenkins-Smith, H., Braman, D., (2011). Cultural cognition of scientific consensus. Journal of Risk Research, 14, 147-174.

Kapucu, N. (2005). Interorganizational coordination in dynamic context: networks in emergencyresponse management. Connections, 26, 35-50.

Kapucu, N., Garayev, V. (2011). Collaborative Decision-Making in Emergency and Disaster Management, International Journal of Public Administration, 34 (6), 366-375.

Kinnear, S., Patison, K., Mann, J., Malone, E., Ross, V. (2014). Network governance and climate change adaptation: Collaborative responses to the Queensland floods. National Climate Change Adaptation Facility. Gold Coast.

Kostoulas, D., Aldunate, R., Pena Mora, F., Lakhera, S. (2008). A nature-inspired decentralized trust model to reduce information unreliability in complex disaster relief operations. Advanced Engineering Informatics, 22, 45-58.

Kramer D., M. (1999). Trust and distrust in organisations: Emerging perspectives, enduring questions. Annual Review of Psychology, 50, 569-99.

Lebel, L. (2014). Closing knowledge-action gaps in adaptation to climate change in the AsiaPacific region, International Journal of Environment and Sustainable Development, 13 (2), 204-222.

Longstaff, P. H., Yang, S. (2008). Communication management and trust: their role in building resilience to "surprises" such as natural disasters, pandemic flu, and terrorism. Ecology and Society, 13(1): 3.

Malone, E., Kinnear, S. (2014). How and why: complementary analyses of social network structures and cultural values: improving flood response networks in Queensland, Australia. Quality and Quantity 1-18.

Marar, Z. (2003). The happiness paradox. Reaktion: London.

McEvily, B., Perrone, V., Zaheer, A. (2003). Trust as an organizing principle.

Organization Science, 14, 91-103.

Moe, T., L., Pathranarakul, P. (2006). An integrated approach to natural disaster management: Public project management and its critical success factors, Disaster Prevention and Management, 15 (3), 396-413.

Nakagawa, Y., Shaw, R. (2004). Social capital: a missing link to disaster recovery. International Journal of Mass Emergencies and Disasters, 22, (1), 5-34.

Nooteboom, B. (2002). Trust: Forms, Foundations, Functions, Failures and Figures. Edward Elgar: Cheltenham, UK and Northampton, MA, USA.

Ospina, A., V. (2011). The role of trust in climate change adaptation and resilience: can ICTs help? Nexus for ICTs, climate change and development (NICCD). 
http://niccd.wordpress.com/2011/02/27/the-role-of-trust-in-climate-change-adaptation-andresilience

Palttala, P., Boano, C., Lund, R., Vos, M. (2012). Communication Gaps in Disaster Management: Perceptions by Experts from Governmental and Non-Governmental Organizations. Journal of Contingencies and Crisis Management, 20, 2-12.

Provan, K. G., Kenis, P. (2008). Modes of Network Governance: Structure, Management, and Effectiveness. Journal of Public Administration Research and Theory, 18 (2), 229-52.

Putnam, R., D. (1993). Making Democracy Work: Civic Traditions in Modern Italy. Princeton University Press: Princeton, NJ.

Putnam, R., D. (1995). Bowling alone: America's declining social capital. Journal of Democracy, 6, 65-78.

Ross, V.L., Fielding, K.S., Louis, W.R. (2014). Social trust, risk perceptions and public acceptance of recycled water: Testing a social-psychological model. Journal of Environmental Management, 137, 61-68.

Rousseau, D.M., Sitkin, S.B., Burt, R.S., Camerer, C. (1998). Not so different after all: a cross discipline view of trust. Academy of Management Review, 23, 393-404.

Seeger, M., W. (2006). Best Practices in Crisis Communication: An Expert Panel Process, Journal of Applied Communication Research, 34 (3), 232-244.

Siegrist, M., Earle, T.C., Gutscher, H. (2007). Trust in Cooperative Risk Management. Earthscan: London.

Tajfel, H., Turner, J.C. (1986). The social identity theory of inter-group behaviour. In: Worchel, S., Austin, L.W. (Eds.), Psychology of Intergroup Relations. Nelson-Hall, Chigago.

Tanis, M., Postmes, T. (2005). A social identity approach to trust: interpersonal perception, group membership and trusting behaviour. European Journal of Social Psychology, 35, 413424.

Tummers, L., Steijn, B., Bekkers, V. (2012) Explaining the Willingness of Public Professionals to Implement Public Policies: Content, Context and Personality Characteristics. Public Administration, 90 (3), 716-36.

Twyman, M., Harvey, N., Harries, C. (2008). Trust in motives, trust in competence: Separate factors determining the effectiveness of risk communication. Judgment and Decision Making, 3, 111-120.

Tyler, T., R., Degoey, P. (1995). Collective restraint in social dilemmas: Procedural justice and social identification effects on support for authorities. Journal of Personality and Social Psychology, 69, 482-97.

Tyler,T., R., Degoey, P. (1996). Trust in organizational authorities. In: R. M. Kramer; T.R. Tyler, (Eds), Trust in organizations. Frontiers of theory and research. London: Sage. 
Vasavada, T. (2013). Managing Disaster Networks in India, Public Management Review, 15 (3), 363-382.

Vaske, J.J., Bright, A.D., Absher, J.D. (2007). Salient value similarity, social trust, and attitudes toward wildland fire management strategies. Human Ecology Review, 14 (2), 223232.

Vaughan, G.M., Hogg, M.A. (1995). Introduction to Social Psychology, 4th Edition ed. Prentice Hall: Sydney.

World Meteorological Organization (WMO). Retrieved 1/07/2014 http://www.wmo.int/pages/prog/drr/documents/Climate_DRR-FactSheet_EN.pdf 